\title{
Systematic Review \\ Prenatal Mercury Exposure and Neurodevelopment up to the Age of 5 Years: A Systematic Review
}

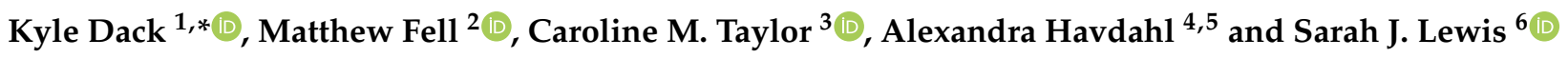 \\ 1 Medical Research Council Integrative Epidemiology Unit, University of Bristol, Bristol BS8 1TH, UK \\ 2 Cleft Collective, University of Bristol, Bristol BS8 1TH, UK; mattfell@doctors.org.uk \\ 3 Centre for Academic Child Health, Bristol Medical School, University of Bristol, Bristol BS8 1TH, UK; \\ Caroline.m.taylor@bristol.ac.uk \\ 4 Department of Mental Disorders, Norwegian Institute of Public Health, 0213 Oslo, Norway; \\ Alexandra.havdahl@fhi.no \\ 5 Nic Waals Institute, Lovisenberg Diaconal Hospital, 0853 Oslo, Norway \\ 6 Population Health Sciences, Bristol Medical School, University of Bristol, Bristol BS8 1TH, UK; \\ s.j.lewis@bristol.ac.uk \\ * Correspondence: Kyle.Dack@Bristol.ac.uk
}

check for

updates

Citation: Dack, K.; Fell, M.; Taylor,

C.M.; Havdahl, A.; Lewis, S.J.

Prenatal Mercury Exposure and

Neurodevelopment up to the Age of

5 Years: A Systematic Review. Int. J.

Environ. Res. Public Health 2022, 19,

1976. https://doi.org/10.3390/

ijerph19041976

Academic Editor: Paul B.

Tchounwou

Received: 30 October 2021

Accepted: 25 January 2022

Published: 10 February 2022

Publisher's Note: MDPI stays neutral with regard to jurisdictional claims in published maps and institutional affiliations.

Copyright: () 2022 by the authors. Licensee MDPI, Basel, Switzerland. This article is an open access article distributed under the terms and conditions of the Creative Commons Attribution (CC BY) license (https:// creativecommons.org/licenses/by/ $4.0 /)$.

\begin{abstract}
Neurodevelopmental delays can interfere with children's engagement with the world and further development, and may have negative consequences into adulthood. Mercury is highly toxic and may negatively influence neurodevelopment because it can freely cross the placenta and accumulate in the fetal brain. We searched four publication databases (Embase, PsycINFO, PubMed/MEDLINE, Scopus) for studies examining the relationship between early life mercury exposure and scores on neurodevelopmental performance measures in children aged 0 to 5 years old. Study quality was assessed using the National Institutes of Health (NIH) Quality Assessment Tool. Thirty-two prospective studies were included in the review. Neurodevelopmental performance was measured using 23 different scales, most commonly the Bayley Scales of Infant and Toddler Development (BSID). In most cases, the evidence for an association between mercury and neurodevelopment was weak. There did not appear to be exceptions for particular childhood ages, outcome scales, or mercury levels. The small number of results to the contrary were more likely to be studies which did not meet our high-quality criteria, and could be a consequence of multiple testing, selection bias, or incomplete confounder adjustment. Based on current evidence, dietary mercury exposure during pregnancy is unlikely to be a risk factor for low neurodevelopmental functioning in early childhood.
\end{abstract}

Keywords: systematic review; pregnancy; childhood; mercury; methylmercury; neurodevelopment

\section{Introduction}

During gestation and infancy, the brain undergoes rapid cell specialization and the shaping of neuronal networks which support neurodevelopmental functions such as cognitive, motor, language, and social skills [1]. Neurodevelopmental difficulties in early life may restrict a child's range of experiences and lead to a cycle of impaired development that results in long-term harm to health and life opportunities [2,3]. For example, a child with motor difficulties may lose opportunities to play with peers, which then restricts the range of experiences and may affect social development. The prenatal environment is known to be a key factor in enabling optimal neurodevelopment [4], and exposure to toxic metals may play a role.

One such element is mercury $(\mathrm{Hg})$. Direct contact with mercury (such as the inhalation of mercury vapors or skin contact) is extremely toxic and can result in organ failure and permanent damage throughout the central nervous system $[5,6]$. This is because mercury is highly reactive and disrupts cellular functions including protein and enzyme inhibition, epigenetic modifications, oxidative stress, and cellular death [7]. Mercury is deposited 
into all major oceans, primarily from industrial atmospheric emissions [8], where a cyclical process of methylation into organic $\mathrm{Hg}$ in the form of methylmercury (MeHg) occurs $[9,10]$. Aquatic MeHg can enter the human food chain following absorption by microorganisms and bioaccumulation into large fish such as tuna and swordfish which may be consumed by humans $[8,11]$. This dietary exposure is likely to increase in coming decades because of the decade-long time lag between atmospheric mercury emissions and ocean absorption, which means that that ocean $\mathrm{Hg}$ is expected to increase regardless of reductions in atmospheric emissions [8,12,13].

Methylmercury is the most common form of dietary $\mathrm{Hg}$ and is highly absorbable in the intestines [14,15], after which it can cross both the placenta [16] and blood-brain barrier $[17,18]$. Hg concentrations are higher in the neonatal umbilical cord than maternal blood [17,19], indicating that the fetus may be exposed to a significant proportion of the mother's circulating mercury. Harmful concentrations may accumulate $[20,21]$ during a critical window of neurological development [22], because the developing infant has both higher levels of mercury crossing the blood-brain barrier and less efficient clearance than adults $[7,23,24]$.

Several epidemiological studies investigated prenatal mercury exposure and neurodevelopment. A review in 2018 [23] identified 11 studies which found evidence of neurodevelopmental harm from mercury exposure during pregnancy. A neurotoxicological review in the same year by Olivera et al. [7] concluded that the evidence from observational studies remained unclear. Neither of the reviews were systematic, and they did not evaluate study quality or compare study methodologies, which may have led to poor quality studies biasing their findings. A 2017 systematic review and meta-analysis of case-control studies compared mercury levels between individuals diagnosed with autism, a neurodevelopmental condition characterized by social communication impairments and repetitive behavior patterns, and typically developing individuals, and concluded that mercury causally increased the likelihood of autism [25]. However, this analysis did not consider potential reverse causation (e.g., autism sensory-seeking symptoms leading to higher mercury exposure), or which covariates were adjusted for in the analysis, an important omission given that there is the potential for confounding from factors such as fish intake [26]. Fish are a source of both methylmercury and of essential nutrients hypothesized to be involved in reducing the likelihood of autism such as long-chain polyunsaturated fatty acids (LCPUFA) $[27,28]$.

This study aims to review the evidence concerning prenatal mercury exposure and early neurodevelopment systematically. Specific objectives are: (1) to identify systematically studies of mercury concentrations in maternal biomarkers and neurodevelopmental functioning in children aged 0 to 5 years old; (2) to assess the quality of studies; (3) to synthesize all results and identify the current pattern and strength of evidence.

\section{Materials and Methods}

\subsection{Study Design and Protocol}

This systematic review was designed to assess the evidence for an association between prenatal exposure to mercury and neurodevelopment in early childhood ( $0-5$ years) from studies published up to December 2020. The age range of 0-5 years was selected because the first years of infancy are understood to form a critical window of brain development [29], and the clearance rate of mercury and methylmercury is such that prenatal exposure would not be expected to last beyond the first few years of life [30,31]. Studies which used biological samples to measure mercury concentrations during pregnancy or at delivery were included; ethylmercury was not included because the pathways to exposure and biological mechanisms are different from other forms of mercury. Studies were included which evaluated neurodevelopmental functioning using continuous scales (not neurodevelopmental diagnoses or symptom measures). The study design and protocol were registered with the International Prospective Register of Systematic Reviews (PROSPERO) on 23 November 
2020, registration number CRD42020221146. Changes to the study and rationale are stated in Supplementary File Part 1.

\subsection{Search Strategy}

We identified search terms and subject headings (where possible) related to pregnancy, mercury, and neurodevelopment.

Search queries were developed for four bibliographic databases: Embase, MEDLINE (PubMed), PsycINFO, and Scopus. Animal studies were excluded, but there was no restriction by year of publication or language. Search queries are available in Supplementary File Part 2. We used Google search to identify relevant white papers, theses, and conference proceedings, using combinations of search terms and screening the first 5 pages of results. The reference list of each included paper was screened to identify additional studies missed by our database searches. The searches were run on 1 December 2020.

\subsection{Study Selection}

Search results were imported into Covidence systematic review software and automatically deduplicated [32]. Papers were screened in a two-stage process by K.D. and M.F. First, titles and abstracts were screened against our inclusion and exclusion criteria in Table 1. Second, papers which passed the first stage of screening were read in full, and a final decision was made by each reviewer. When studies were regarded as eligible by one reviewer but not the other, the studies were discussed to come to a consensus decision.

Table 1. Criteria for including or excluding papers from this systematic review.

\begin{tabular}{ll}
\hline \multicolumn{1}{c}{ Include } & \multicolumn{1}{c}{ Exclude } \\
\hline $\begin{array}{l}\text { 1. Study of total mercury, inorganic, organic, or } \\
\text { methylmercury compounds. }\end{array}$ & 1. Studies other compounds including ethylmercury. \\
\hline 2. Measures mercury in pregnant women. & 2. Measures mercury in other populations. \\
\hline $\begin{array}{l}\text { 3. Measures mercury concentrations in biological samples: blood } \\
\text { (whole, erythrocyte, plasma, serum), urine, cord blood/tissue, } \\
\text { placenta, or hair. }\end{array}$ & 3. Uses any other measure of mercury exposure. \\
\hline $\begin{array}{l}\text { 4. Measures neurodevelopmental functioning (i.e.: cognition, } \\
\text { attention, memory, intelligence, fine/gross motor development, } \\
\text { receptive/expressive language ability, communicative ability, social } \\
\text { development, or overall neurodevelopment) in children aged 0 to }\end{array}$ & $\begin{array}{l}\text { 4. Measures neurodevelopmental functioning in older } \\
\text { shildren or adults, or only measures diagnoses or } \\
\text { syears old. }\end{array}$ \\
\hline $\begin{array}{l}\text { 5. Reports association between mercury and neurodevelopmental disorders. } \\
\text { neurodevelopmental functioning }\end{array}$ & $\begin{array}{l}\text { 5. Does not report associations between mercury and } \\
\text { specified outcomes. }\end{array}$ \\
\hline $\begin{array}{l}\text { 6. Study reports results from multivariable analysis methods. } \\
\text { 6. Study reports results only from univariable methods } \\
\text { such as correlations or t-tests. }\end{array}$ \\
\hline
\end{tabular}

\subsection{Data Extraction and Quality Assessment}

The following data were extracted: Publication details, study type, location, mercury measurement method, neurodevelopmental functioning measurement tool, population, statistical methods, model adjustment variables, and results. A full list of data fields is shown in Supplementary File Part 3. The extracted data were checked by the second reviewer.

The quality of each study was assessed using the National Institutes of Health (NIH) Quality Assessment Tool [33], which was designed for prospective and cross-sectional studies (Supplementary File Part 4). The tool evaluates the design, sampling, measurement, and reporting quality of studies using 14 yes/no questions. Two further items were added which we judged important to consider: (1) whether studies selected covariates based on significance testing, which may encourage bias compared to selection based on prior evidence and theory [34]; (2) whether studies fully published the results of their planned analyses, or only those which met a significance threshold. 
Studies were considered high quality if: (a) they met 12 or more of the 16 quality assessment criteria; and (b) they met item 14 of the NIH Quality Assessment Tool: "Were key potential confounding variables measured and adjusted statistically for their impact on the relationship between exposure(s) and outcome(s)?". Item 14 was considered essential because the failure to adjust for important confounders would lead to bias in the model estimates. We identified (1) maternal socio-economic status or education, (2) fish or fatty acid intake, and (3) maternal smoking as key confounders [35-41].

\subsection{Evidence Synthesis}

We could not meta-analyze study results because of the numerous sources of heterogeneity in the design of studies, including mercury measurement, sample type, neurodevelopmental domain and assessment tool, the timing of exposure and outcome measurements, and type of statistical estimate. We instead synthesized results in a narrative review.

Studies used a variety of neurodevelopmental scales many of which measured different domains of neurodevelopmental functioning. We grouped the review into six broad domains which the neurodevelopmental outcomes could be mapped to:

1. Cognition and language as measured by the Bayley Scales of Infant and Toddler Development II (BSID-II) Mental Developmental Index (MDI).

2. Other measure of cognition, including attention, executive function, and memory.

3. Motor function, fine, and gross.

4. Communication and language development.

5. Social development.

6. General or composite measures of neurodevelopmental functioning.

\section{Results}

\subsection{Study Characteristics}

The search returned 593 potential studies after deduplication, and 6 more were found among reference lists. See Figure 1 for numbers at each stage of screening. We found 32 studies eligible for inclusion in this review, listed in Appendix A.

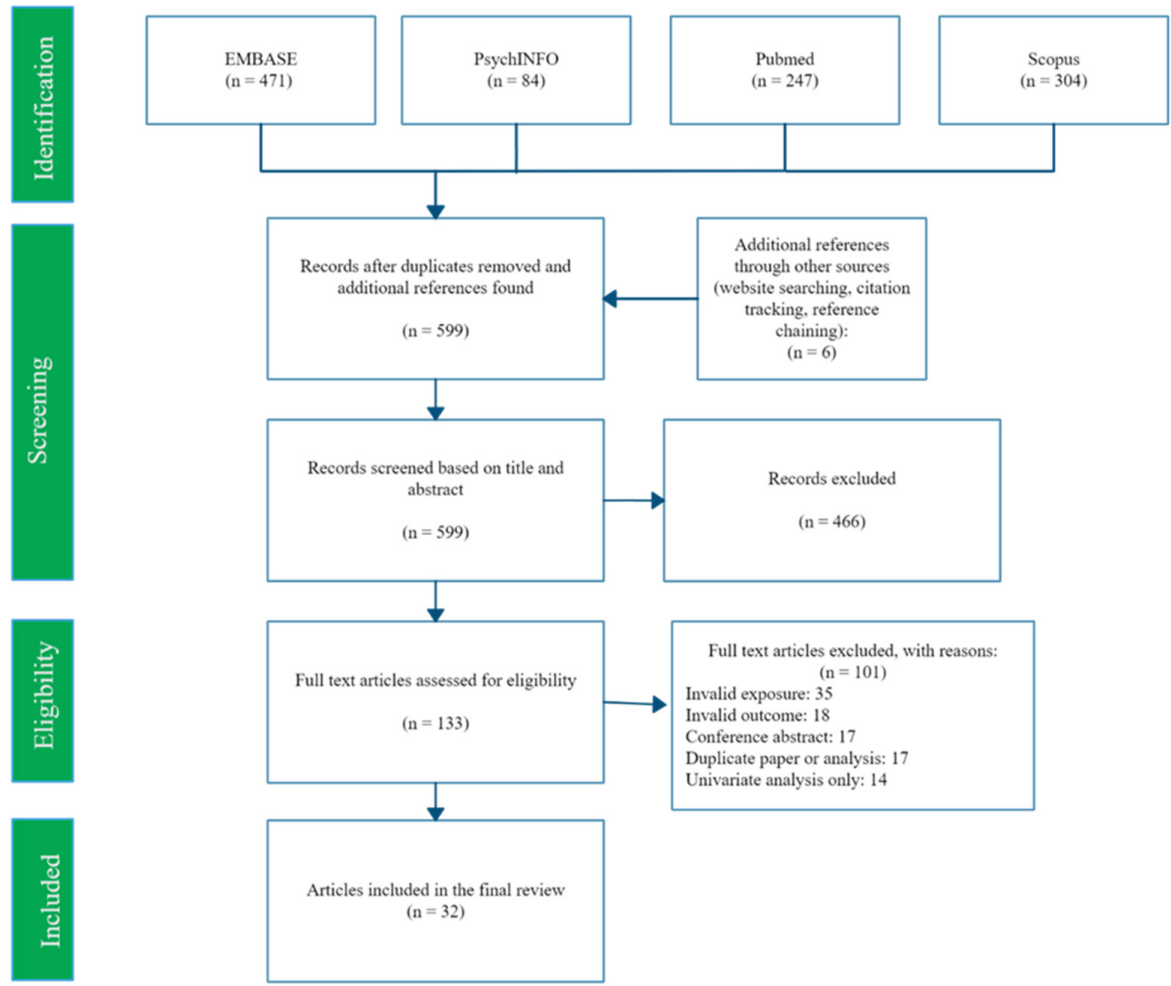

Figure 1. PRISMA flow diagram of search and selection process. 
Neurodevelopmental functioning was measured using 23 scales, and the most common were variations of the BSID scale, found in 17 studies (Table 2). The BSID-II and Bayley-III) (also referred to as BSID-III) were designed to measure cognitive, motor, and language development in children aged 1 to 42 months. Scales are standardized across all ages to a mean of 100 and standard deviation of 15 . The two versions are reviewed separately because performance on the two versions is correlated but not identical [42], and motor and language development is measured in a single scale in the second edition but not the third. Further scale details are available in Supplementary File Part 5.

Table 2. Measures of neurodevelopmental functioning in the included studies.

\begin{tabular}{|c|c|c|}
\hline Abbreviation & Name & $n$ \\
\hline A-not-B & A-not-B test & 1 \\
\hline BSID-II & Bayley Scales of Infant and Toddler Development-Second Edition & 9 \\
\hline Bayley-III/BSID-III & Bayley Scales of Infant and Toddler Development-Third Edition & 8 \\
\hline CDI & MacArthur-Bates Communicative Development Inventories & 1 \\
\hline CDIIT & Comprehensive Developmental Inventory for Infants and Toddlers & 1 \\
\hline DDST & Denver Developmental Screening Test & 1 \\
\hline DDST (modified) & Modified version of the Denver Developmental Screening Test & 1 \\
\hline FTII & Fagan Test of Infant Intelligence & 1 \\
\hline GDS & Gesell developmental schedules & 2 \\
\hline $\mathrm{K}-\mathrm{ABC}$ & Kaufman Assessment Battery for Children & 1 \\
\hline K-BSID-II & $\begin{array}{l}\text { Korean adapted version of Bayley Scales of Infant and Toddler } \\
\text { Development II }\end{array}$ & 1 \\
\hline KSPD & Kyoto Scale of Psychological Development & 1 \\
\hline MCDI & MacArthur Communicative Development Inventory & 1 \\
\hline MDAT & Malawi Developmental Assessment Tool & 1 \\
\hline MSCA & McCarthy Scales of Children's Abilities & 1 \\
\hline NBAS & Neonatal Behavioral Assessment Scale & 1 \\
\hline NBNA & Neonatal Behavioral Neurological Assessment & 2 \\
\hline NNNS & NICU Network Neurobehavioral Scale & 1 \\
\hline PPVT & Peabody Picture Vocabulary Test & 1 \\
\hline SMS & Social Maturity Scale (Vineland) & 1 \\
\hline VRM & Visual recognition memory & 1 \\
\hline WPPSI-R & Wechsler Preschool and Primary Scales of Intelligence-Revised & 2 \\
\hline WRAVMA & Wide Range Assessment of Visual Motor Abilities & 1 \\
\hline
\end{tabular}

All used a prospective design where tissue samples were taken prior to neurodevelopmental assessment, most frequently from the mother during pregnancy or delivery (Table 3$)$. Tissue types commonly used to measure mercury concentrations were maternal whole blood $(n=10)$, hair $(n=10)$, or umbilical cord blood or tissue $(n=21)$. The studies took place in 16 countries where the primary source of mercury exposure was mostly fish or seafood consumption (e.g., [43]), but also from rice consumption [44], and proximity to artisanal tin [45] or gold mining [46]. 
Table 3. Study characteristics.

\begin{tabular}{|c|c|c|c|}
\hline Country & $n^{1}$ & & $n^{1}$ \\
\hline Brazil & 1 & Republic of Seychelles & 2 \\
\hline Canada & 1 & Slovenia & 2 \\
\hline China & 4 & South Korea & 3 \\
\hline Croatia & 4 & Spain & 2 \\
\hline Greece & 1 & Taiwan & 1 \\
\hline Italy & 4 & Tanzania & 1 \\
\hline Japan & 3 & United Kingdom & 2 \\
\hline Poland & 2 & USA & 4 \\
\hline Mercury source (all maternal) & $n$ & Mercury analysis & $n$ \\
\hline Whole blood & 10 & Atomic absorption spectroscopy (AAS) & 3 \\
\hline Erythrocyte & 1 & $\begin{array}{c}\text { Cold vapour atomic absorption } \\
\text { spectrometry (CVAAS) }\end{array}$ & 22 \\
\hline Hair & 10 & $\begin{array}{l}\text { Inductively coupled plasma mass } \\
\text { spectrometry (ICP-DRC-MS) }\end{array}$ & 1 \\
\hline Placenta & 1 & $\begin{array}{l}\text { Inductively coupled plasma mass } \\
\text { spectrometry (ICP-MS) }\end{array}$ & 4 \\
\hline Umbilical cord & 21 & & \\
\hline
\end{tabular}

${ }^{1}$ Studies may be counted more than once if they include more than one country, mercury source, or mercury analysis method.

Where reported, we recorded central tendency measures of mercury concentrations which are shown in Supplementary File Part 6. The mean Hg in maternal whole blood ranged from 0.64 [47] to $3.71 \mu \mathrm{g} / \mathrm{L}$ [48], and maternal hair sample concentrations from 0.3 [49] to $5.7 \mu \mathrm{g} / \mathrm{g}$ [50]. Not all umbilical cord blood or tissue concentrations were reported in comparable units, but one Taiwanese cohort had a mean cord blood $\mathrm{Hg}$ of $14.9 \mu \mathrm{g} / \mathrm{L}$ which was considerably higher than other studies [51].

Studies frequently did not meet NIH Quality Assessment criteria concerning transparency and reporting (results in Supplementary File Part 7). Most studies did not state their initial recruitment participation rate (item 3), and 5 did not explain why they recruited the reported sample size (item 5). Most studies either did not report their follow-up rate, or their loss to follow-up was greater than $20 \%$ of the initial population (item 13). Only 15 studies adjusted for all key confounders (item 14), and it was common for studies instead to select covariates using significance testing ( $n=10$, item 15$)$. Overall, 15 studies passed our criteria for high quality.

\subsection{Evidence Summary}

This review contains 32 studies. Many of the studies included associations between mercury and both composite scores and subscales from neurodevelopmental assessment tools in their main results. It was common for studies to measure mercury concentrations in more than one tissue type and assess neurodevelopment at more than one time point (e.g., 12 months, 24 months). For these reasons there were many more results than studies: 175 estimates (median 4.5). Tables 4-9 below include only studies which met our highquality criteria, with results from all studies available in Supplementary File Part 8. 
Table 4. Results from studies identified as high quality which measured cognition and language in the BSID:II MDI scale $(n=4)$.

\begin{tabular}{|c|c|c|c|c|c|c|c|c|}
\hline Study & $n$ & Exposure & Units & Outcome & $\begin{array}{l}\text { Time of } \\
\text { Outcome }\end{array}$ & Estimate & $95 \% \mathrm{CI}$ & $p$-Value \\
\hline Boucher et al. (2014) & 87 & Umbilical cord & $\mathrm{Ln} \mu \mathrm{g} / \mathrm{L}$ & BSID-II: MDI & 11 months & 0.08 & -0.15 to 0.33 & \\
\hline Kim Y et al. (2018) & 595 & Umbilical cord & $\mu g / L$ & BSID-II: MDI & 12 months & -0.04 & -0.39 to 0.32 & 0.85 \\
\hline Kim Y et al. (2018) & 523 & Umbilical cord & $\mu \mathrm{g} / \mathrm{L}$ & BSID-II: MDI & 24 months & -0.03 & -0.36 to 0.3 & 0.87 \\
\hline Kim Y et al. (2018) & 438 & Umbilical cord & $\mu \mathrm{g} / \mathrm{L}$ & BSID-II: MDI & 36 months & 0.17 & -0.26 to 0.6 & 0.43 \\
\hline Kim Y et al. (2018) & 662 & Umbilical cord & $\mu \mathrm{g} / \mathrm{L}$ & BSID-II: MDI & 6 months & -0.03 & -0.27 to 0.21 & 0.8 \\
\hline Kim Y et al. (2018) & 763 & Whole blood (early pregnancy) & $\mu \mathrm{g} / \mathrm{L}$ & BSID-II: MDI & 12 months & -0.32 & -0.89 to 0.26 & 0.28 \\
\hline Kim Y et al. (2018) & 614 & Whole blood (late pregnancy) & $\mu \mathrm{g} / \mathrm{L}$ & BSID-II: MDI & 12 months & -0.07 & -0.69 to 0.55 & 0.82 \\
\hline Kim Y et al. (2018) & 564 & Whole blood (late pregnancy) & $\mu \mathrm{g} / \mathrm{L}$ & BSID-II: MDI & 24 months & -0.46 & -1.03 to 0.12 & 0.12 \\
\hline Kim Y et al. (2018) & 557 & Whole blood (early pregnancy) & $\mu \mathrm{g} / \mathrm{L}$ & BSID-II: MDI & 36 months & -0.28 & -0.89 to 0.32 & 0.36 \\
\hline Kim Y et al. (2018) & 460 & Whole blood (late pregnancy) & $\mu \mathrm{g} / \mathrm{L}$ & BSID-II: MDI & 36 months & -0.25 & -0.88 to 0.38 & 0.43 \\
\hline Kim Y et al. (2018) & 847 & Whole blood (early pregnancy) & $\mu \mathrm{g} / \mathrm{L}$ & BSID-II: MDI & 6 months & -0.41 & -0.81 to -0.003 & 0.048 \\
\hline Kim Y et al. (2018) & 689 & Whole blood (late pregnancy) & $\mu \mathrm{g} / \mathrm{L}$ & BSID-II: MDI & 6 months & -0.13 & -0.56 to 0.29 & 0.54 \\
\hline Llop et al. (2012) & 1683 & Umbilical cord & $\mathrm{Ln} \mu \mathrm{g} / \mathrm{L}$ & BSID-II: MDI & 14 months & 0.16 & -0.12 to 0.45 & \\
\hline $\begin{array}{l}\text { Rothenberg et al. } \\
\text { (2016) }\end{array}$ & 270 & Hair & Ln $\mu \mathrm{g} / \mathrm{g}$ & BSID-II: MDI & 12 months & -4.9 & -9.7 to -0.1 & \\
\hline
\end{tabular}


Table 5. Results from studies identified as high quality which measured cognition $(n=7)$.

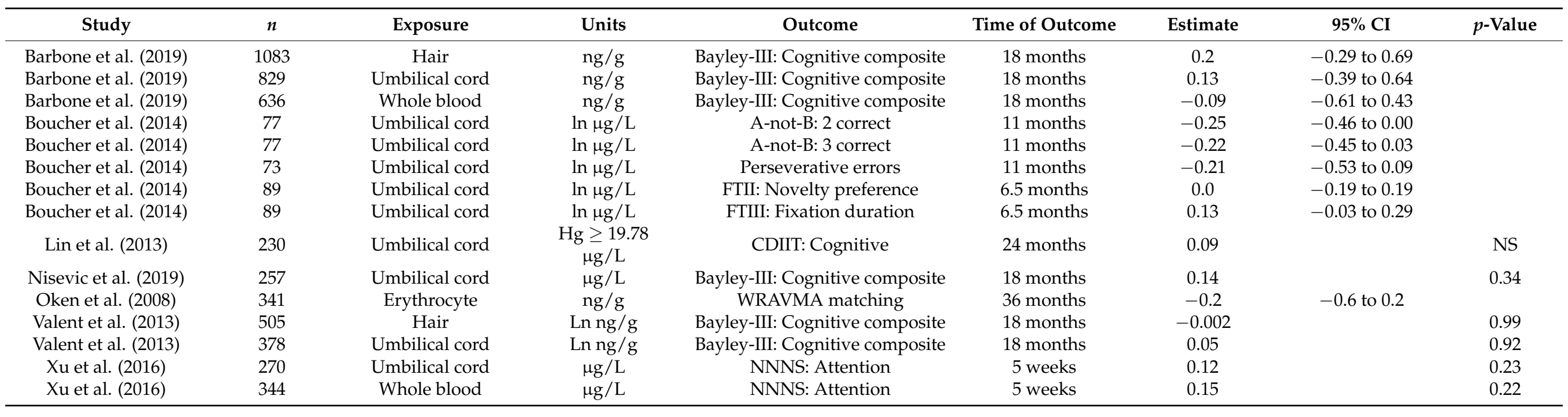


Table 6. Results from studies identified as high quality which measured motor function $(n=11)$.

\begin{tabular}{|c|c|c|c|c|c|c|c|c|}
\hline Study & $n$ & Exposure & Units & Outcome & Time of Outcome & Estimate & $95 \% \mathrm{CI}$ & $p$-Value \\
\hline Barbone et al. (2019) & 1082 & Hair & $\mathrm{ng} / \mathrm{g}$ & Bayley-III: Fine Motor scale & 18 months & -0.03 & -0.11 to 0.06 & \\
\hline Barbone et al. (2019) & 1081 & Hair & $\mathrm{ng} / \mathrm{g}$ & Bayley-III: Gross Motor scale & 18 months & -0.01 & -0.07 to 0.05 & \\
\hline Barbone et al. (2019) & 1083 & Hair & $\mathrm{ng} / \mathrm{g}$ & Bayley-III: Motor development & 18 months & -0.12 & -0.47 to 0.22 & \\
\hline Barbone et al. (2019) & 892 & Umbilical cord & $\mathrm{ng} / \mathrm{g}$ & Bayley-III: Motor development & 18 months & -0.11 & -0.47 to 0.25 & \\
\hline Barbone et al. (2019) & 636 & Whole blood & $\mathrm{ng} / \mathrm{g}$ & Bayley-III: Fine Motor scale & 18 months & 0.05 & -0.04 to 0.15 & \\
\hline Barbone et al. (2019) & 890 & Whole blood & $\mathrm{ng} / \mathrm{g}$ & Bayley-III: Gross Motor scale & 18 months & -0.03 & -0.09 to 0.03 & \\
\hline Barbone et al. (2019) & 636 & Whole blood & $\mathrm{ng} / \mathrm{g}$ & Bayley-III: Motor development & 18 months & 0.11 & -0.25 to 0.48 & \\
\hline Hu et al. (2016) & 410 & Umbilical cord & $\operatorname{Ln} \mu g / L$ & GDS: Fine motor domain & 12 months & -2.62 & -7.78 to 2.55 & \\
\hline Hu et al. (2016) & 410 & Umbilical cord & $\mathrm{Ln} \mu \mathrm{g} / \mathrm{L}$ & GDS: Gross motor domain & 12 months & 1.95 & -3.08 to 6.98 & \\
\hline Hu et al. (2016) & 410 & Whole blood & $\mathrm{Ln} \mu \mathrm{g} / \mathrm{L}$ & GDS: Fine motor domain & 12 months & 2.69 & -3.37 to 8.74 & \\
\hline Hu et al. (2016) & 410 & Whole blood & $\mathrm{Ln} \mu \mathrm{g} / \mathrm{L}$ & GDS: Gross motor domain & 12 months & 3.26 & -2.72 to 9.24 & \\
\hline Kim Y et al. (2018) & 595 & Umbilical cord & $\mu \mathrm{g} / \mathrm{L}$ & BSID-II: PDI & 12 months & 0.14 & -0.23 to 0.52 & 0.45 \\
\hline Kim Y et al. (2018) & 523 & Umbilical cord & $\mu \mathrm{g} / \mathrm{L}$ & BSID-II: PDI & 24 months & 0.16 & -0.17 to 0.48 & 0.34 \\
\hline Kim Y et al. (2018) & 438 & Umbilical cord & $\mu \mathrm{g} / \mathrm{L}$ & BSID-II: PDI & 36 months & -0.13 & -0.55 to 0.27 & 0.53 \\
\hline Kim Y et al. (2018) & 662 & Umbilical cord & $\mu \mathrm{g} / \mathrm{L}$ & BSID-II: PDI & 6 months & -0.2 & -0.45 to 0.15 & 0.33 \\
\hline Kim Y et al. (2018) & 763 & $\begin{array}{l}\text { Whole blood (early } \\
\text { pregnancy) }\end{array}$ & $\mu \mathrm{g} / \mathrm{L}$ & BSID-II: PDI & 12 months & 0.3 & -0.31 to 0.91 & 0.34 \\
\hline Kim Y et al. (2018) & 614 & $\begin{array}{l}\text { Whole blood (late } \\
\text { pregnancy) }\end{array}$ & $\mu \mathrm{g} / \mathrm{L}$ & BSID-II: PDI & 12 months & 0.27 & -0.38 to 0.93 & 0.41 \\
\hline Kim Y et al. (2018) & 686 & $\begin{array}{c}\text { Whole blood (early } \\
\text { pregnancy) }\end{array}$ & $\mu \mathrm{g} / \mathrm{L}$ & BSID-II: PDI & 24 months & -0.17 & -0.74 to 0.4 & 0.56 \\
\hline Kim Y et al. (2018) & 564 & $\begin{array}{c}\text { Whole blood (late } \\
\text { pregnancy) }\end{array}$ & $\mu \mathrm{g} / \mathrm{L}$ & BSID-II: PDI & 24 months & -0.09 & -0.67 to 0.48 & 0.75 \\
\hline
\end{tabular}


Table 6. Cont.

\begin{tabular}{|c|c|c|c|c|c|c|c|c|}
\hline Study & $n$ & Exposure & Units & Outcome & Time of Outcome & Estimate & $95 \% \mathrm{CI}$ & $p$-Value \\
\hline Kim Y et al. (2018) & 557 & $\begin{array}{c}\text { Whole blood (early } \\
\text { pregnancy) }\end{array}$ & $\mu \mathrm{g} / \mathrm{L}$ & BSID-II: PDI & 36 months & -0.11 & -0.7 to 0.47 & 0.70 \\
\hline Kim Y et al. (2018) & 460 & $\begin{array}{c}\text { Whole blood (late } \\
\text { pregnancy) }\end{array}$ & $\mu \mathrm{g} / \mathrm{L}$ & BSID-II: PDI & 36 months & -0.58 & -1.19 to 0.03 & 0.06 \\
\hline Kim Y et al. (2018) & 847 & $\begin{array}{c}\text { Whole blood (early } \\
\text { pregnancy) }\end{array}$ & $\mu \mathrm{g} / \mathrm{L}$ & BSID-II: PDI & 6 months & -0.55 & -1.05 to -0.05 & 0.03 \\
\hline Kim Y et al. (2018) & 689 & $\begin{array}{l}\text { Whole blood (late } \\
\text { pregnancy) }\end{array}$ & $\mu \mathrm{g} / \mathrm{L}$ & BSID-II: PDI & 6 months & -0.27 & -0.78 to 0.25 & 0.31 \\
\hline Llop et al. (2012) & 1683 & Umbilical cord & $\operatorname{Ln} \mu \mathrm{g} / \mathrm{L}$ & BSID-II: PDI & 14 months & -0.05 & -0.79 to 0.68 & \\
\hline Nisevic et al. (2019) & 257 & Umbilical cord & $\mu \mathrm{g} / \mathrm{L}$ & Bayley-III: Fine Motor scale & 18 months & -0.07 & & 0.78 \\
\hline Nisevic et al. (2019) & 257 & Umbilical cord & $\mu \mathrm{g} / \mathrm{L}$ & Bayley-III: Gross Motor scale & 18 months & 0.08 & & 0.71 \\
\hline Nisevic et al. (2019) & 257 & Umbilical cord & $\mu \mathrm{g} / \mathrm{L}$ & Bayley-III: Motor development & 18 months & 0.01 & & 0.92 \\
\hline Oken et al. (2008) & 341 & Erythrocyte & $\mathrm{ng} / \mathrm{g}$ & WRAVMA drawing & 36 months & 0.1 & -0.2 to 0.4 & \\
\hline Oken et al. (2008) & 341 & Erythrocyte & $\mathrm{ng} / \mathrm{g}$ & WRAVMA pegboard & 36 months & 0.03 & -0.3 to 0.3 & \\
\hline $\begin{array}{l}\text { Rothenberg et al. } \\
\qquad(2016)\end{array}$ & 270 & Hair & $\operatorname{Ln} \mu \mathrm{g} / \mathrm{g}$ & BSID-II: PDI & 12 months & -2.7 & -8.3 to 2.9 & \\
\hline Tatsuta et al. (2017) & 566 & Umbilical cord & Ln ng/g & BSID-II: PDI & 18 months & -0.12 & & 0.009 \\
\hline Valent et al. (2013) & 505 & Hair & Ln ng/g & Bayley-III: Motor development & 18 months & -0.19 & & 0.62 \\
\hline Xu et al. (2016) & 270 & Umbilical cord & $\mu \mathrm{g} / \mathrm{L}$ & NNNS: Asymmetry (Female) & 5 weeks & 0.07 & & 0.4 \\
\hline Xu et al. (2016) & 270 & Umbilical cord & $\mu \mathrm{g} / \mathrm{L}$ & NNNS: Handling & 5 weeks & -0.02 & & 0.35 \\
\hline Xu et al. (2016) & 344 & Whole blood & $\mu \mathrm{g} / \mathrm{L}$ & NNNS: Asymmetry (Male) & 5 weeks & -0.13 & & 0.3 \\
\hline Xu et al. (2016) & 344 & Whole blood (Female) & $\mu \mathrm{g} / \mathrm{L}$ & NNNS: Asymmetry (Female) & 5 weeks & 0.08 & & 0.43 \\
\hline Xu et al. (2016) & 344 & Whole blood & $\mu \mathrm{g} / \mathrm{L}$ & NNNS: Handling & 5 weeks & -0.001 & & 0.98 \\
\hline
\end{tabular}


Table 7. Results from studies identified as high quality which measured communication and language development $(n=6)$.

\begin{tabular}{|c|c|c|c|c|c|c|c|c|}
\hline Study & $n$ & Exposure & Units & Outcome & $\begin{array}{l}\text { Time of } \\
\text { Outcome }\end{array}$ & Estimate & $95 \%$ CI & $p$-Value \\
\hline Barbone et al. (2019) & 1272 & Hair & $\mathrm{ng} / \mathrm{g}$ & $\begin{array}{l}\text { Bayley-III: Expressive } \\
\text { Communication scale }\end{array}$ & 18 months & 0.04 & -0.06 to 0.13 & \\
\hline Barbone et al. (2019) & 1086 & Hair & $\mathrm{ng} / \mathrm{g}$ & $\begin{array}{l}\text { Bayley-III: Language } \\
\text { composite }\end{array}$ & 18 months & 0.55 & 0.05 to 1.05 & \\
\hline Barbone et al. (2019) & 1075 & Hair & $\mathrm{ng} / \mathrm{g}$ & $\begin{array}{l}\text { Bayley-III: Receptive } \\
\text { Communication scale }\end{array}$ & 18 months & 0.12 & 0.02 to 0.22 & \\
\hline Barbone et al. (2019) & 1070 & Umbilical cord & $\mathrm{ng} / \mathrm{g}$ & $\begin{array}{l}\text { Bayley-III: Expressive } \\
\text { Communication scale }\end{array}$ & 18 months & 0.01 & -0.09 to 0.11 & \\
\hline Barbone et al. (2019) & 896 & Umbilical cord & $\mathrm{ng} / \mathrm{g}$ & $\begin{array}{l}\text { Bayley-III: Language } \\
\text { composite }\end{array}$ & 18 months & 0.25 & -0.29 to 0.78 & \\
\hline Barbone et al. (2019) & 887 & Umbilical cord & $\mathrm{ng} / \mathrm{g}$ & $\begin{array}{l}\text { Bayley-III: Receptive } \\
\text { Communication scale }\end{array}$ & 18 months & 0.12 & -0.08 to 0.32 & \\
\hline Barbone et al. (2019) & 727 & Whole blood & $\mathrm{ng} / \mathrm{g}$ & $\begin{array}{l}\text { Bayley-III: Expressive } \\
\text { Communication scale }\end{array}$ & 18 months & 0.13 & -0.22 to 0.48 & \\
\hline Barbone et al. (2019) & 628 & Whole blood & $\mathrm{ng} / \mathrm{g}$ & $\begin{array}{l}\text { Bayley-III: Receptive } \\
\text { Communication scale }\end{array}$ & 18 months & -0.02 & -0.12 to 0.08 & \\
\hline Daniels et al. (2004) & 1054 & Umbilical cord & $\mu \mathrm{g} / \mathrm{g}$ & $\begin{array}{l}\text { MCDI: Vocabulary } \\
\text { Comprehension }\end{array}$ & 15 months & 6.1 & & 0.8 \\
\hline Hu et al. (2016) & 410 & Umbilical cord & $\mathrm{Ln} \mu \mathrm{g} / \mathrm{L}$ & GDS: Language domain & 12 months & 2.17 & -1.88 to 6.21 & \\
\hline Hu et al. (2016) & 410 & Whole blood & $\mathrm{Ln} \mu \mathrm{g} / \mathrm{L}$ & GDS: Language domain & 12 months & 1.92 & -3.61 to 7.46 & \\
\hline Nisevic et al. (2019) & 257 & Umbilical cord & $\mu \mathrm{g} / \mathrm{L}$ & $\begin{array}{l}\text { Bayley-III: Language } \\
\text { composite }\end{array}$ & 18 months & -0.05 & & 0.74 \\
\hline Oken et al. (2008) & 341 & Erythrocyte & $\mathrm{ng} / \mathrm{g}$ & PPVT & 36 months & -0.4 & -0.8 to -0.1 & \\
\hline Valent et al. (2013) & 505 & Hair & Ln ng/g & $\begin{array}{l}\text { Bayley-III: Language } \\
\text { composite }\end{array}$ & 18 months & 0.85 & & 0.11 \\
\hline Valent et al. (2013) & 378 & Umbilical cord & Ln ng/g & $\begin{array}{l}\text { Bayley-III: Language } \\
\text { composite }\end{array}$ & 18 months & 0.41 & & 0.46 \\
\hline
\end{tabular}


Table 8. Results from studies identified as high quality which measured social development $(n=3)$.

\begin{tabular}{|c|c|c|c|c|c|c|c|c|}
\hline Study & $n$ & Exposure & Units & Outcome & Time of Outcome & Estimate & $95 \% \mathrm{CI}$ & $p$-Value \\
\hline Daniels et al. (2004) & 1054 & Umbilical cord & $\mu \mathrm{g} / \mathrm{g}$ & MCDI: Social activity & 15 months & -0.2 & & 0.9 \\
\hline Daniels et al. (2004) & 1054 & Umbilical cord & $\mu \mathrm{g} / \mathrm{g}$ & DDST: Social Activity & 18 months & 0.5 & & 0.8 \\
\hline Hu et al. (2016) & 410 & Umbilical cord & $\mathrm{Ln} \mu \mathrm{g} / \mathrm{L}$ & GDS: Social domain & 12 months & 4.06 & 0.51 to 7.62 & \\
\hline Valent et al. (2013) & 505 & Hair & Ln ng/g & $\begin{array}{c}\text { Bayley-III: } \\
\text { Social-emotional }\end{array}$ & 18 months & 1.77 & & 0.11 \\
\hline Valent et al. (2013) & 378 & Umbilical cord & Ln ng/g & $\begin{array}{c}\text { Bayley-III: } \\
\text { Social-emotional }\end{array}$ & 18 months & -0.07 & & 0.95 \\
\hline
\end{tabular}

Table 9. Results from studies identified as high quality which measured general or composite measures of neurodevelopmental functioning $(n=6)$.

\begin{tabular}{|c|c|c|c|c|c|c|c|c|}
\hline Study & $n$ & Exposure & Units & Outcome & $\begin{array}{l}\text { Time of } \\
\text { Outcome }\end{array}$ & Estimate & $95 \% \mathrm{CI}$ & $p$-Value \\
\hline Daniels et al. (2004) & 1054 & Umbilical cord & $\mu \mathrm{g} / \mathrm{g}$ & DDST: Total & 18 months & 0.4 & & 0.9 \\
\hline Golding et al. (2016) & 2643 & Whole blood & $\mu \mathrm{g} / \mathrm{L}$ & DDST-II & 18 months & 0.49 & 0.1 to 0.88 & 0.01 \\
\hline Golding et al. (2016) & 2394 & Whole blood & $\mu \mathrm{g} / \mathrm{L}$ & DDST-II & 32 months & 0.43 & 0.08 to 0.78 & 0.02 \\
\hline Golding et al. (2016) & 2721 & Whole blood & $\mu \mathrm{g} / \mathrm{L}$ & DDST-II & 6 months & 0.51 & 0.05 to 1 & 0.03 \\
\hline Hu et al. (2016) & 410 & Umbilical cord & $\mathrm{Ln} \mu \mathrm{g} / \mathrm{L}$ & GDS: Adaptive domain & 12 months & 4.22 & 0.77 to 7.67 & \\
\hline Hu et al. (2016) & 410 & Whole blood & $\mathrm{Ln} \mu \mathrm{g} / \mathrm{L}$ & GDS: Adaptive domain & 12 months & 0.65 & -4.3 to 5.59 & \\
\hline Suzuki et al. (2010) & 498 & Hair & $\mu \mathrm{g} / \mathrm{g}$ & NBAS & 3 days & -0.12 & & $<0.05$ \\
\hline Valent et al. (2013) & 362 & Hair & Ln ng/g & $\begin{array}{c}\text { Bayley-III: Adaptive } \\
\text { behaviour }\end{array}$ & 18 months & 0.55 & & 0.56 \\
\hline Valent et al. (2013) & 271 & Umbilical cord & Ln ng/g & $\begin{array}{c}\text { Bayley-III: Adaptive } \\
\text { behaviour }\end{array}$ & 18 months & -0.57 & -0.15 to 0.33 & 0.57 \\
\hline
\end{tabular}




\subsubsection{Cognition and Language as Measured by the BSID-II MDI $(n=10)$}

The BSID-II is a standardized developmental test for infants and toddlers [52] and measures both cognitive and language development. It comprises the Psychomotor Development Index (PDI-reviewed later) and the MDI. We found 10 studies of maternal mercury $\mathrm{Hg}$ and childhood MDI, and the range of sample sizes was 87 to 1683 . Whilst most observed negative associations, in almost all cases the confidence interval overlapped with the null and indicated only weak evidence of an association.

The pattern of results was consistent between studies using maternal whole blood, hair, and umbilical cord samples. Two South Korean studies using maternal whole blood found no strong evidence of an association in samples taken in both the first and third trimesters of pregnancy $[48,53]$. Similarly, weak evidence of negative associations was reported in studies of maternal hair mercury [50,54]. However, one study of $\mathrm{MeHg}$ exposure through rice consumption in China did identify stronger evidence albeit with wide confidence intervals ( $-4.9 \mathrm{MDI}$ points per $\log \mu \mathrm{g} / \mathrm{g}, 95 \% \mathrm{CI}:-9.7$ to -0.1$)$ [44].

Seven studies used umbilical cord blood or tissue [48,53,55-59]. One of those studies reported a negative association but did not adjust for fish or LCPUFA intake during pregnancy; the other studies did not find strong evidence of an association.

From the 10 studies of MDI, 4 were high quality [44,48,55,59]. Increased mercury exposure tended to be associated with lower MDI in these studies, but only one study reported an association which did not overlap with the null [44]. MDI represents both cognition and language development, but specific measures of cognition and language are reviewed in Sections 2 and 4.

3.2.2. Other Measure of Cognition, Including Attention, Executive Function, and Memory $(n=13)$

The most common method used to measure cognition was the Bayley-III cognitive composite. This is different to the MDI of BSID-II because it is designed to measure cognitive performance more specifically, and includes the extreme ends of cognitive performance [60]. It was used in 7 studies of children in populations aged 12-40 months, none of which reported strong evidence of a negative association with maternal mercury concentrations $[49,61-66]$. This includes three studies which met our criteria for high quality, including an adjustment for key confounders [61,63,65].

Three studies used other measures of general cognition in cohorts that the authors hypothesized were exposed to high levels of mercury. Two cohorts of Japanese children at 42 months [67] and Taiwanese children aged 24 months [51] both suggested mercury exposure had occurred through seafood consumption, but neither reported strong evidence of an association between cord blood mercury concentrations and overall cognition. A US study of pregnant women in New York identified both seafood and air particles following the World Trade Center disaster as possible routes of exposure. It reported strong evidence of negative associations between umbilical cord mercury and full, performance, and verbal IQ scores in children aged 4 years old [58].

Memory [68], executive function [68], attention [47], visual matching [69], visual recognition [70], or object permanence and novelty fixation [55] were the subject of five studies of children at various stages of infancy up to 3 years old. In most cases, no strong evidence of an association was reported. However, performance on A-not-B tasks, which are intended to reflect object permanence, was negatively associated with cord $\mathrm{Hg}$ in one study [55]. Although confidence intervals overlapped with the null ( -0.25 A-not-B 2 correct, 95\% CI: -0.46 to $0.00 ;-0.22$ A-not-B 3 correct, CI: -0.45 to $0.03 ;-0.21$ perseverative errors score, CI: -0.53 to 0.09 ), this is likely because of small sample sizes in the three analyses $(n=73-79)$.

Overall, the 13 studies described in this section did not find strong evidence of an association. This finding was consistent in six studies identified as high quality $[47,51,61,63,65,69]$. A seventh high quality but small study $(n=73-79)$ also did not report strong evidence of an 
association, but as discussed in the previous paragraph appeared to be underpowered [55]. The source and level of mercury exposure varied but did not affect the pattern of results.

\subsubsection{Motor Function, Fine, and Gross $(n=23)$}

Ten studies used the BSID-II PDI to measure motor function in populations aged from 6 to 36 months $[44,48,50,53-56,58,59,71]$. The estimated association with maternal Hg was close to zero with no strong evidence of an association reported in five studies [53,54]three of which were high quality $[44,55,59]$. Four other studies of maternal $\mathrm{Hg}$ reported 15 negative associations with PDI and 5 positive, but only 5 estimates-all negative-did not overlap with the null $[48,50,56,58]$, and only one study was high quality [48]. While one further study reported evidence of a negative association $(-0.119$ PDI per Ln $\mathrm{ng} / \mathrm{g}$ $\mathrm{Hg}, p=0.009$ ) with umbilical cord mercury concentrations and PDI [71], this study may be affected by reporting bias because it did not report all the results of its planned analyses.

Seven studies used the Bayley-III motor development scale, all including children of a roughly similar age (12 to 24 months) [49,61,63-66,72]. Maternal hair and umbilical cord sample mercury concentrations were in most cases found to be positively associated with overall motor development, but with confidence intervals overlapping the null in all studies $[49,61,63,65,66]$. The only strong evidence was a negative association reported between umbilical cord sample $\mathrm{Hg}$ and fine motor development [64], a finding not replicated in other studies of this sub-scale [61,63,72]. In studies using other measures of motor development, none reported strong evidence of an association with maternal mercury concentrations $[46,47,51,68,69,73]$.

Of the six studies of motor development which reported evidence for a negative association, none adjusted for fish or fatty acid consumption. In the 11 studies which met our criteria for high quality, the pattern of evidence was different, with almost all results reporting no strong evidence of an association. In these high-quality studies, most appeared adequately powered based on their effect sizes and confidence intervals, but two which reported non-significant results had quite wide $95 \%$ confidence intervals which may indicate a lack of power [44,73]. Other factors such as study size or tissue sampled did not differ between studies marked high or not high quality.

In summary, there was little indication that mercury was associated with motor development. Unlike MDI or cognition where negative but non-significant point estimates were often found, for motor development there was no such pattern, and most estimates were close to zero.

\subsubsection{Communication and Language Development $(n=11)$}

Six studies using the Bayley-III language composite scale reported no strong evidence of an association at 12-24 months with maternal hair or umbilical cord mercury [49,61,63-66].

Two studies reported negative associations between maternal blood $\mathrm{Hg}$ and Peabody Picture Vocabulary performance [69] and The Malawi Developmental Assessment Tool language scale [46]. One of these met our high-quality criteria and measured second trimester erythrocyte concentrations, which primarily consist of $\mathrm{MeHg}$ [69]. Three other studies used umbilical cord samples, including one of 1054 children, and each reported no evidence of an association with language development $[51,73,74]$.

In the six studies identified as high quality $[61,63,65,69,73,74]$, most estimates were positive and overlapped the null. Low power did not appear to be an issue in most of these studies because estimates were close to the null without wide confidence intervals or relatively small sample sizes, although one study did appear to be underpowered (2.17 GDS language domain per $\mathrm{Ln} \mu \mathrm{g} / \mathrm{L}$ umbilical cord $\mathrm{Hg},-1.88$ to 6.21) [73].

\subsubsection{Social Development $(n=5)$}

Five studies measured social development in children aged between 6 and 18 months, three of which were high quality. No strong evidence of an association was found with maternal whole blood $\mathrm{Hg}[46,73]$, hair $\mathrm{Hg}$ [65], or umbilical cord samples [51,65,73,74]. 
Three studies were high quality $[65,73,74]$. One high-quality study contained wide confidence intervals which indicates possible low power; this study observed a positive effect (0.74 Gesell Developmental Schedules social domain score per $\mathrm{Ln} \mu \mathrm{g} / \mathrm{L},-5.77$ to 4.31 , $n=410)$ [73].

\subsubsection{General or Composite Measures of Neurodevelopmental Functioning $(n=11)$}

Eleven studies used scales of neurodevelopment designed to assess overall functioning or could not otherwise be classified. Three of these were of neonatal functional ability and reflexes at 3 days old, two of which reported strong evidence of a negative association with mercury concentrations in maternal hair [43] and umbilical cord samples [66] taken at delivery. The third reported a positive association with umbilical cord Hg [75]. The two studies using umbilical cord samples both met 12 or more NIH quality criteria and adjusted for key confounders.

Six studies looked at mercury concentrations and general neurodevelopmental functioning during childhood $[45,46,51,69,74,76]$. One reported evidence of a negative association between maternal mercury and neurodevelopmental functioning [46]. However, this did not adjust for key confounding variables. Three studies met our high-quality criteria and reported either no evidence of an association $[69,74]$ or strong evidence of a positive association in both fish eaters and non-fish eaters [76].

Two studies measured adaptive functioning, which encompasses the functional use of neurodevelopmental skills in daily life. One reported no strong evidence of an association [65], while the other found strong evidence of a positive association with umbilical cord $\mathrm{Hg}$ [73], which is the opposite direction to what would be expected if a neurotoxic effect was present. Both studies were high quality and adjusted for fish consumption. The latter suggested that despite adjustment for fish consumption, $\mathrm{Hg}$ levels may have been too low to overcome the benefits of fish intake (geometric mean of maternal blood $\mathrm{Hg}$ : $0.72 \mu \mathrm{g} / \mathrm{L}$, IQR: 0.54 to $1.05 \mu \mathrm{g} / \mathrm{L})$.

\section{Discussion}

This systematic review found 32 studies of childhood neurodevelopmental functioning in children aged from 3 days to 59 months old. The most widely used methods of measurement were the 2 nd and 3rd versions of the BSID. Nineteen other measures were also used. Mercury concentrations were measured in tissues taken during pregnancy or at delivery.

The evidence for an association between mercury exposure and neurodevelopmental functioning was weak. While there were 17 results that provided evidence of a negative relationship, there were also 8 which indicated evidence of a positive association, and a further 150 where the estimated association overlapped with the null. We examined patterns of results by mercury biomarker, the timing of measurement, and child age, and the lack of evidence was consistent in all cases. Some authors suggested that whilst their findings were not indicative of a negative association, there may have been an age band where the neurological effects of mercury may be more clearly detectable. However, we did not identify stronger evidence at a particular age band. While three studies of neonatal behavior all reported evidence of an association $[43,66,75]$ with maternal hair or umbilical cord mercury concentrations, the reliability of the neurological assessment of neonates may be less than that of older children. Neonates have a limited range of behaviors available, and there is a heightened role of the assessor in interpreting behavior, which may increase the risk of measurement error.

Fifteen high-quality studies of children aged 0 to 36 months were identified using a modified NIH QA tool. These studies were less likely to report strong evidence of an association, which may indicate where negative associations were reported; it may have been a consequence of study bias. While most studies appeared to be adequately powered, it is possible that five were underpowered because they reported wide confidence intervals which overlapped with the null $[44,53,56,67,73]$. 
The theorized adverse effects of mercury on neurodevelopment may be more detectable above a certain threshold of exposure. First, current measures of neurodevelopment may be unable to detect the small changes that occur at lower levels of exposure, which could still translate to significant adverse effects on a population level. Secondly, at lower levels there may adequate levels of nutrients which protect against oxidative stress [77] or aid mercury excretion [78]. This review included studies with mean mercury concentrations from 0.64 to $3.71 \mu \mathrm{g} / \mathrm{L}$ for whole blood samples, and 0.3 to $5.7 \mu \mathrm{g} / \mathrm{g}$ for maternal hair (Supplementary File Part 6). The findings of this review may not be applicable to populations with higher levels of mercury exposure, such as those that were reported in studies of the First Nations people in Canada [79] and the Faroe Islands [80].

There are few guidelines which consider a safe limit or reference value for circulating mercury during pregnancy and fetal health. The German FEA reported a reference value of $2.0 \mu \mathrm{g} / \mathrm{L}$ blood $\mathrm{Hg}$ for all adults [81]. The US EPA (2003) used data from three studies in the Faroe Islands, Seychelles Islands, and New Zealand to calculate a reference dose of blood $\mathrm{Hg}$ below which neurotoxicity is unlikely [82]. However, this estimate was based on multiple pharmacokinetic assumptions, and the studies themselves were not consistent in finding a threshold effect [83]. A US cohort not included in the EPA analysis estimated a reference dose level of $3.5 \mu \mathrm{g} / \mathrm{L}$ to avoid risks to the fetal nervous system [84]. Each of these estimates is towards the higher end of the distributions reported by studies in this review. The WHO suggested a safe limit for hair $\mathrm{Hg}$ of $10 \mathrm{ng} / \mathrm{g}$ [85] which is also above the mean from many studies in this review. Further guidelines exist in the context of fish consumption [86] which cannot easily be translated to circulating $\mathrm{Hg}$ concentrations. If neurotoxic effects from mercury are more detectable at higher levels of exposure, this does not appear to have been studied within the age range and other criteria included in our literature search.

Our findings do not replicate those of a systematic review of maternal dietary mercury intake which included 15 studies of neurodevelopmental outcomes in children up to 8 years old [87]. The review concluded there was clear evidence that maternal dietary exposure adversely affected childhood cognitive development, although this may be mitigated by nutrients in mercury-containing foods such as fish. Seven studies from this review were not included in our own because: they were carried out in children over 5 years of age, reported only univariate results, or duplicated an analysis contained in another included paper. This review included 17 studies which were not in the earlier review, most likely because the studies did not focus on dietary mercury exposure, and this may be a reason for the difference in our conclusions. A systematic review of fish consumption during pregnancy and childhood neurodevelopment concluded there was moderate evidence of a beneficial effect [88]. Our finding that there is a lack of evidence for a negative association between mercury concentrations and early childhood neurodevelopment is consistent with this, but not directly comparable.

There are several limitations to our review. First, it includes studies using a wide variety of neurodevelopmental measures, which measure different domains of neurodevelopmental functioning, and some measures are not directly comparable. To address this, we grouped and summarized results by the aspect of neurodevelopment being measured, such as cognition or motor function. However, these groupings are approximate generalizations, and there may be alternative methods of summarizing the results which more accurately represent the underlying neurodevelopmental dimensions. Additionally, we did not appraise the ability of the scales to represent neurodevelopmental functioning accurately in young children, and it is unlikely that all measures used in our included studies are equally valid. Finally, this review did not include studies which measured neurodevelopmental condition diagnoses or symptoms. It may be that different results are seen in those outcomes.

The second limitation of the review is the assumption that mercury concentrations in maternal whole blood, hair, placenta, and umbilical cord samples are correlated with prenatal mercury exposure. While the intercorrelation between these biomarkers is well 
established (e.g., $[89,90])$, there is limited evidence to confirm the hypothesized correlation with mercury concentrations in the fetus itself [16]. It may be that maternal blood or hair mercury correlates poorly with fetal mercury exposure, which would affect the inclusion criteria of this review. Thirdly, the high degree of heterogeneity in the methods of included studies meant it was not possible to synthesize results in a quantitative manner such as through meta-analysis. Finally, it is possible that despite finding limited evidence of an association between $\mathrm{Hg}$ and neurodevelopment, many studies included in the review were small and underpowered to detect more modest effects. Therefore, there may still be a small adverse relationship which would translate to an important deficit in, for example, intellectual capital, in a large population.

This review's strengths are first that we conducted a literature search on a wide range of neurodevelopmental functions in infants and young children, and were able to find a large number of studies. This enabled us to compare the pattern of results along many study dimensions such as the characteristics of study populations and the exposure and outcome measurement timing and methods. Our findings were consistent across these study characteristics, but there were indications of heterogeneity. Some studies reported positive associations while others were negative, and there were a minority of studies reporting strong evidence of an association in both directions. Secondly, the review was conducted in a systematic way, with the results and conclusions based on all the reported results in the included studies. This may give a more accurate and comprehensive overview of the evidence compared to previous narrative reviews which tended to discuss only significant results. Third, we conducted the review in a transparent manner by following a registered protocol and publishing all data used to inform the review. Finally, we identified high-quality studies using an externally developed quality assessment tool and by examining the specific model parameterization used in each study.

\section{Conclusions}

At the levels of mercury recorded in studies included in this review, the evidence for an association between prenatal mercury concentrations and neurodevelopmental functioning in children from 0 to 5 years is weak. No pattern was identified by the age of child or study methodology. Any adverse effect may also be too small to be clinically detectable. Fish contains many essential nutrients involved in brain development such as LCPUFA, so where fish is the main source of dietary $\mathrm{Hg}$, these other nutrients may compensate against the toxic effects of mercury. Future studies may wish to focus on populations with higher levels of mercury exposure or consider alternative study designs with different assumptions and limitations, such as natural experiments or genetic analyses.

Supplementary Materials: The following are available online at https:/ /www.mdpi.com/article/10 .3390/ijerph19041976/s1, Part 1: Changes to the protocol, Part 2: Search terms, Part 3: fields used in data extraction, Part 4: Quality assessment tool, Part 5: Measures of neurodevelopmental functioning in the included studies, Part 6: Mercury exposure characteristics in included studies, Part 7: Results from NIH Quality Assessment, Part 8: Results from all studies.

Author Contributions: Conceptualization, K.D. and S.J.L.; methodology, K.D. and S.J.L.; software, K.D. and M.F.; validation, K.D. and M.F.; formal analysis, K.D. and M.F.; investigation, K.D. and M.F.; data curation, K.D.; writing—original draft preparation, K.D.; writing—review and editing, M.F., C.M.T., A.H. and S.J.L.; visualization, K.D. and S.J.L.; supervision, S.J.L. and C.M.T. All authors have read and agreed to the published version of the manuscript. 
Funding: This research was funded by the following sources. K.D. is supported by a Ph.D. studentship from the MRC Integrative Epidemiology Unit at the University of Bristol (faculty matched place for MRC and Peter and Jean James Scholarship). M.F. received funding from the VTCT Foundation for a research fellowship with the Cleft Collective at the University of Bristol. C.M.T. is supported by a MRC Career Development Award (MR/T010010/1). A.H. is supported by a career grant from the South-Eastern Norway Regional Health Authority (2020022) and the Research Council of Norway (274611, 288083). S.J.L. is supported by the NIHR Bio-medical Research Centre at University Hospitals Bristol and Weston NHS Foundation Trust and the University of Bristol. Publication was supported by MRC IEU grant MC_UU_00011/1.

Institutional Review Board Statement: Not applicable.

Informed Consent Statement: Not applicable.

Data Availability Statement: All extracted data used to write this review are included in Appendix A and Supplementary File Parts 1-8.

Conflicts of Interest: The authors declare no conflict of interest. The funders had no role in the design of the study; in the collection, analyses, or interpretation of data; in the writing of the manuscript; or in the decision to publish the results. 


\section{Appendix A. Included Studies}

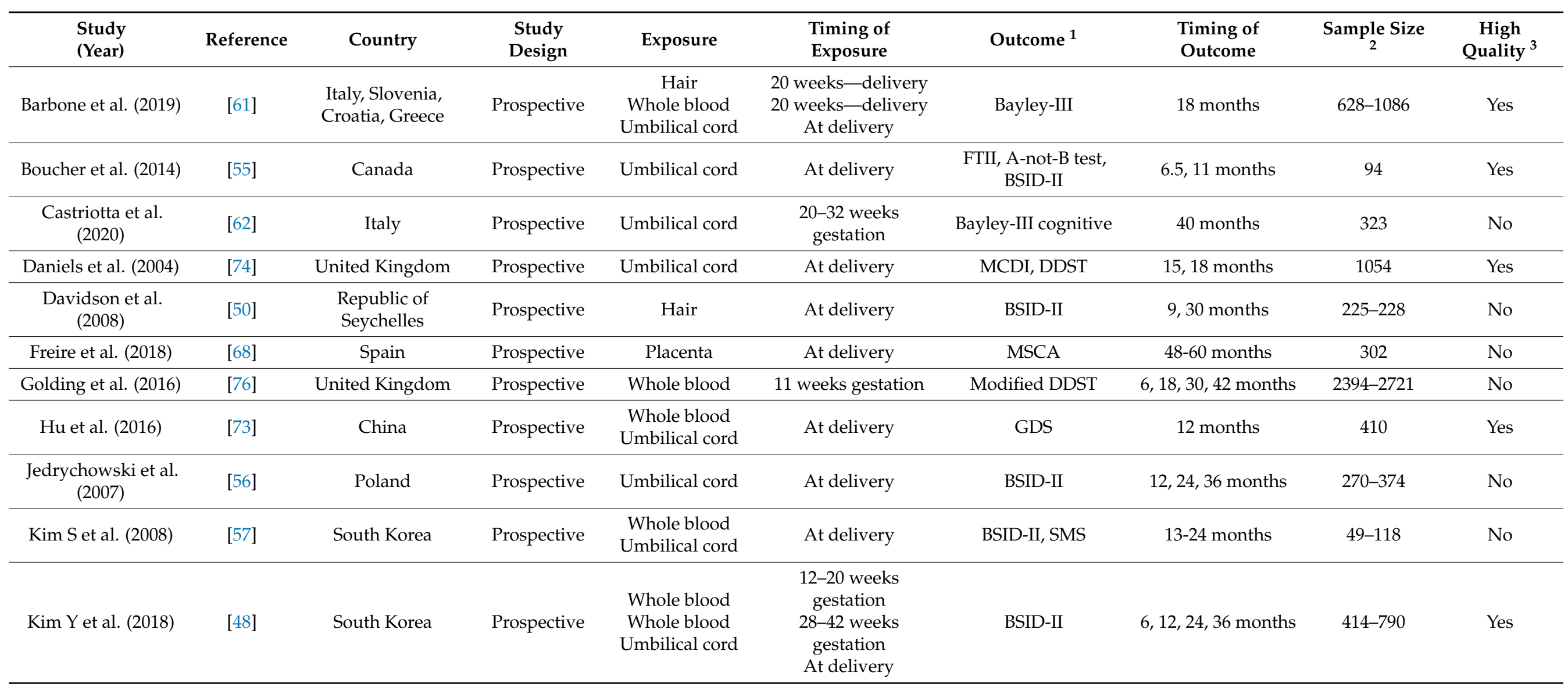




\begin{tabular}{|c|c|c|c|c|c|c|c|c|c|}
\hline $\begin{array}{l}\text { Study } \\
\text { (Year) }\end{array}$ & Reference & Country & $\begin{array}{l}\text { Study } \\
\text { Design }\end{array}$ & Exposure & $\begin{array}{l}\text { Timing of } \\
\text { Exposure }\end{array}$ & Outcome $^{1}$ & $\begin{array}{l}\text { Timing of } \\
\text { Outcome }\end{array}$ & $\underset{2}{\text { Sample Size }}$ & $\begin{array}{c}\text { High } \\
\text { Quality }^{3}\end{array}$ \\
\hline $\begin{array}{l}\text { Lederman et al. } \\
\qquad(2008)\end{array}$ & [58] & USA & Prospective & Umbilical cord & At delivery & BSID-II, WPPSI-R & $12,24,36,48$ months & $107-132$ & No \\
\hline Lin et al. (2013) & & Taiwan & Prospective & Umbilical cord & At delivery & CDIIT & 24 months & 230 & No \\
\hline Llop et al. (2012) & [59] & Spain & Prospective & Umbilical cord & At delivery & BSID-II & 14 months & 1683 & Yes \\
\hline $\begin{array}{l}\text { Marques et al. } \\
\text { (2009) }\end{array}$ & [45] & Brazil & Prospective & Hair & At delivery & GDS & $6,36,60$ months & 82 & No \\
\hline Nisevic et al. (2019) & [63] & Croatia, Italy & Prospective & Umbilical cord & At delivery & Bayley-III & 18 months & 257 & Yes \\
\hline Nyanza et al. (2020) & {$[46]$} & Tanzania & Prospective & Whole blood & $\begin{array}{l}\text { 16-27 weeks } \\
\text { gestation }\end{array}$ & MDAT & 6-12 months & 429 & No \\
\hline Oken et al. (2005) & {$[70]$} & USA & Prospective & Hair & At delivery & VRM & 6 months & 135 & No \\
\hline Oken et al. (2008) & [69] & USA & Prospective & Erythrocyte & 2nd trimester & PPVT, WRAVMA & 36 months & 341 & Yes \\
\hline $\begin{array}{l}\text { Polanska et al. } \\
\text { (2013) }\end{array}$ & [49] & Poland & Prospective & Hair & $\begin{array}{c}\text { 30-34 weeks } \\
\text { gestation }\end{array}$ & Bayley-III & 12-24 months & 303 & No \\
\hline $\begin{array}{l}\text { Rothenberg et al. } \\
\text { (2016) }\end{array}$ & [44] & China & Prospective & $\begin{array}{l}\text { Whole blood } \\
\text { Hair }\end{array}$ & At delivery & BSID-II & 12 months & 270 & Yes \\
\hline $\begin{array}{l}\text { Shah-Kulkarni et al. } \\
\qquad(2020)\end{array}$ & [53] & South Korea & Prospective & $\begin{array}{l}\text { Whole blood } \\
\text { Whole blood } \\
\text { Umbilical cord }\end{array}$ & $\begin{array}{l}\text { 12-20 weeks } \\
\text { gestation } \\
>28 \text { weeks } \\
\text { gestation } \\
\text { At delivery }\end{array}$ & K-BSID-II & 6 months & $321-467$ & No \\
\hline $\begin{array}{l}\text { SnojTratnik et al. } \\
\text { (2017) }\end{array}$ & [64] & Slovenia, Croatia & Prospective & $\begin{array}{c}\text { Hair } \\
\text { Umbilical cord }\end{array}$ & $\begin{array}{c}34 \text { weeks } \\
\text { gestation/at } \\
\text { delivery } \\
\text { At delivery }\end{array}$ & Bayley-III & 16-20 months & $280-357$ & No \\
\hline
\end{tabular}




\begin{tabular}{|c|c|c|c|c|c|c|c|c|c|}
\hline $\begin{array}{l}\text { Study } \\
\text { (Year) }\end{array}$ & Reference & Country & $\begin{array}{c}\text { Study } \\
\text { Design }\end{array}$ & Exposure & $\begin{array}{l}\text { Timing of } \\
\text { Exposure }\end{array}$ & Outcome $^{1}$ & $\begin{array}{l}\text { Timing of } \\
\text { Outcome }\end{array}$ & $\underset{2}{\text { Sample Size }}$ & $\begin{array}{c}\text { High } \\
\text { Quality }^{3}\end{array}$ \\
\hline Strain et al. (2015) & [54] & $\begin{array}{l}\text { Republic of } \\
\text { Seychelles }\end{array}$ & Prospective & Hair & At delivery & BSID-II, CDI & 20 months & $1241-1265$ & No \\
\hline Suzuki et al. (2010) & [43] & Japan & Prospective & Hair & At delivery & NBAS & 3 days & 498 & Yes \\
\hline Tatsuta et al. (2014) & [67] & Japan & Prospective & Umbilical cord & At delivery & $\mathrm{K}-\mathrm{ABC}$ & 42 months & 287 & No \\
\hline Tatsuta et al. (2017) & [71] & Japan & Prospective & Umbilical cord & At delivery & BSID-II, KSPD & 18 months & 566 & Yes \\
\hline Valent et al. (2013) & [65] & Italy & Prospective & $\begin{array}{c}\text { Hair } \\
\text { Umbilical cord }\end{array}$ & $\begin{array}{c}\text { 20-22 weeks } \\
\text { gestationAt delivery }\end{array}$ & Bayley-III & 18 months & $271-505$ & Yes \\
\hline Wang et al. (2019) & [66] & China & Prospective & Umbilical cord & At delivery & $\begin{array}{c}\text { NBNA } \\
\text { Bayley-III }\end{array}$ & 3 days 18 months & $172-265$ & No \\
\hline Wu et al. (2014) & [75] & China & Prospective & Umbilical cord & At delivery & NBNA & 3 days & 418 & No \\
\hline Xu et al. (2016) & [47] & USA & Prospective & $\begin{array}{l}\text { Whole blood } \\
\text { Umbilical cord }\end{array}$ & $\begin{array}{c}16 \text { weeks-delivery } \\
\text { At delivery }\end{array}$ & NNNS & 5 weeks & 344 & Yes \\
\hline
\end{tabular}

${ }^{1}$ For explanation of abbreviations, see Table $2 .{ }^{2}$ Size of primary analysis, or a range if there were multiple primary analyses. ${ }^{3} 12$ or more quality assessment criteria met, including adjustment for key confounders. 


\section{References}

1. Tierney, A.L.; Nelson, C.A., 3rd. Brain Development and the Role of Experience in the Early Years. Zero Three 2009, 30, 9-13. [PubMed]

2. Cusick, S.E.; Georgieff, M.K. The Role of Nutrition in Brain Development: The Golden Opportunity of the "First 1000 Days". J. Pediatr. 2016, 175, 16-21. [CrossRef] [PubMed]

3. Walker, S.P.; Wachs, T.D.; Meeks Gardner, J.; Lozoff, B.; Wasserman, G.A.; Pollitt, E.; Carter, J.A. Child development: Risk factors for adverse outcomes in developing countries. Lancet 2007, 369, 145-157. [CrossRef]

4. Fitzgerald, E.; Hor, K.; Drake, A.J. Maternal influences on fetal brain development: The role of nutrition, infection and stress, and the potential for intergenerational consequences. Early Hum. Dev. 2020, 150, 105190. [CrossRef]

5. Bernhoft, R.A. Mercury toxicity and treatment: A review of the literature. J. Environ. Public Health 2012, 2012, 460508. [CrossRef]

6. Syversen, T.; Kaur, P. The toxicology of mercury and its compounds. J. Trace Elem. Med. Biol. 2012, 26, 215-226. [CrossRef]

7. Oliveira, C.S.; Nogara, P.A.; Ardisson-Araújo, D.M.P.; Aschner, M.; Rocha, J.B.T.; Dórea, J.G. Neurodevelopmental Effects of Mercury. Adv. Neurotoxicol. 2018, 2, 27-86. [CrossRef]

8. Mason, R.P.; Choi, A.L.; Fitzgerald, W.F.; Hammerschmidt, C.R.; Lamborg, C.H.; Soerensen, A.L.; Sunderland, E.M. Mercury biogeochemical cycling in the ocean and policy implications. Environ. Res. 2012, 119, 101-117. [CrossRef]

9. Lin, H.; Ascher, D.B.; Myung, Y.; Lamborg, C.H.; Hallam, S.J.; Gionfriddo, C.M.; Holt, K.E.; Moreau, J.W. Mercury methylation by metabolically versatile and cosmopolitan marine bacteria. ISME J. 2021, 15, 1810-1825. [CrossRef] [PubMed]

10. Munson, K.M.; Lamborg, C.H.; Boiteau, R.M.; Saito, M.A. Dynamic mercury methylation and demethylation in oligotrophic marine water. Biogeosciences 2018, 15, 6451-6460. [CrossRef]

11. Balshaw, S.; Edwards, J.; Daughtry, B.; Ross, K. Mercury in seafood: Mechanisms of accumulation and consequences for consumer health. Rev. Environ. Health 2007, 22, 91-113. [CrossRef] [PubMed]

12. United Nations Environment Programme. Global Mercury Assessment 2018; UN: Geneva, Switzerland, 2019.

13. Sunderland, E.M.; Krabbenhoft, D.P.; Moreau, J.W.; Strode, S.A.; Landing, W.M. Mercury sources, distribution, and bioavailability in the North Pacific Ocean: Insights from data and models. Glob. Biogeochem. Cycles 2009, 23, GB2010. [CrossRef]

14. Hong, Y.-S.; Kim, Y.-M.; Lee, K.-E. Methylmercury exposure and health effects. J. Prev. Med. Public Health 2012, 45, 353-363. [CrossRef]

15. Bradley, M.A.; Barst, B.D.; Basu, N. A Review of Mercury Bioavailability in Humans and Fish. Int. J. Environ. Res. Public Health 2017, 14, 169. [CrossRef]

16. Tong, M.; Yu, J.; Liu, M.; Li, Z.; Wang, L.; Yin, C.; Ren, A.; Chen, L.; Jin, L. Total mercury concentration in placental tissue, a good biomarker of prenatal mercury exposure, is associated with risk for neural tube defects in offspring. Environ. Int. 2021, 150, 106425. [CrossRef]

17. Chen, Z.; Myers, R.; Wei, T.; Bind, E.; Kassim, P.; Wang, G.; Ji, Y.; Hong, X.; Caruso, D.; Bartell, T.; et al. Placental transfer and concentrations of cadmium, mercury, lead, and selenium in mothers, newborns, and young children. J. Expo. Sci. Environ. Epidemiol. 2014, 24, 537-544. [CrossRef] [PubMed]

18. Aschner, M.; Aschner, J.L. Mercury neurotoxicity: Mechanisms of blood-brain barrier transport. Neurosci. Biobehav. Rev. 1990, 14, 169-176. [CrossRef]

19. Sakamoto, M.; Chan, H.M.; Domingo, J.L.; Koriyama, C.; Murata, K. Placental transfer and levels of mercury, selenium, vitamin E, and docosahexaenoic acid in maternal and umbilical cord blood. Environ. Int. 2018, 111, 309-315. [CrossRef]

20. Aberg, B.; Ekman, L.; Falk, R.; Greitz, U.; Persson, G.; Snihs, J.O. Metabolism of Methyl Mercury $\left({ }^{203} \mathrm{Hg}\right)$ Compounds in Man. Arch. Environ. Health Int. J. 1969, 19, 478-484. [CrossRef]

21. Jo, S.; Woo, H.D.; Kwon, H.-J.; Oh, S.-Y.; Park, J.-D.; Hong, Y.-S.; Pyo, H.; Park, K.S.; Ha, M.; Kim, H.; et al. Estimation of the Biological Half-Life of Methylmercury Using a Population Toxicokinetic Model. Int. J. Environ. Res. Public Health 2015, $12,9054$. [CrossRef]

22. Vohr, B.R.; Poggi Davis, E.; Wanke, C.A.; Krebs, N.F. Neurodevelopment: The Impact of Nutrition and Inflammation During Preconception and Pregnancy in Low-Resource Settings. Pediatrics 2017, 139, S38. [CrossRef] [PubMed]

23. Bellinger, D.C. Environmental chemical exposures and neurodevelopmental impairments in children. Pediatr. Med. 2018, 1, 1-13. [CrossRef]

24. Rand, M.D.; Caito, S.W. Variation in the biological half-life of methylmercury in humans: Methods, measurements and meaning. Biochim. Biophys. Acta (BBA) Gen. Subj. 2019, 1863, 129301. [CrossRef] [PubMed]

25. Jafari, T.; Rostampour, N.; Fallah, A.A.; Hesami, A. The association between mercury levels and autism spectrum disorders: A systematic review and meta-analysis. J. Trace Elem. Med. Biol. 2017, 44, 289-297. [CrossRef]

26. Choi, A.L.; Cordier, S.; Weihe, P.; Grandjean, P. Negative confounding in the evaluation of toxicity: The case of methylmercury in fish and seafood. Crit. Rev. Toxicol. 2008, 38, 877-893. [CrossRef]

27. Mazahery, H.; Stonehouse, W.; Delshad, M.; Kruger, M.C.; Conlon, C.A.; Beck, K.L.; von Hurst, P.R. Relationship between Long Chain n-3 Polyunsaturated Fatty Acids and Autism Spectrum Disorder: Systematic Review and Meta-Analysis of Case-Control and Randomised Controlled Trials. Nutrients 2017, 9, 155. [CrossRef]

28. Madore, C.; Leyrolle, Q.; Lacabanne, C.; Benmamar-Badel, A.; Joffre, C.; Nadjar, A.; Layé, S. Neuroinflammation in Autism: Plausible Role of Maternal Inflammation, Dietary Omega 3, and Microbiota. Neural Plast. 2016, 2016, 3597209. [CrossRef] 
29. Scott, J.A. The first 1000 days: A critical period of nutritional opportunity and vulnerability. Nutr. Diet. 2020, $77,295-297$. [CrossRef]

30. Holmes, P.; James, K.A.; Levy, L.S. Is low-level environmental mercury exposure of concern to human health? Sci. Total Environ. 2009, 408, 2. [CrossRef]

31. Clarkson, T.W.; Jayesh, V.B.; Ballatori, N. Mechanisms of mercury disposition in the body. Am. J. Ind. Med. 2007, 50, 10. [CrossRef]

32. Persson, M.; Fagt, S.; Nauta, M.J. Personalised fish intake recommendations: The effect of background exposure on optimisation. Br. J. Nutr. 2018, 120, 946-957. [CrossRef]

33. Quality Assessment Tool for Observational Cohort and Cross-Sectional Studies. Available online: https://www.nhlbi.nih.gov/ health-topics / study-quality-assessment-tools (accessed on 10 March 2021).

34. Lederer, D.J.; Bell, S.C.; Branson, R.D.; Chalmers, J.D.; Marshall, R.; Maslove, D.M.; Ost, D.E.; Punjabi, N.M.; Schatz, M.; Smyth, A.R.; et al. Control of Confounding and Reporting of Results in Causal Inference Studies. Guidance for Authors from Editors of Respiratory, Sleep, and Critical Care Journals. Ann. Am. Thorac. Soc. 2018, 16, 22-28. [CrossRef] [PubMed]

35. Chin-Lun Hung, G.; Hahn, J.; Alamiri, B.; Buka, S.L.; Goldstein, J.M.; Laird, N.; Nelson, C.A.; Smoller, J.W.; Gilman, S.E. Socioeconomic disadvantage and neural development from infancy through early childhood. Int. J. Epidemiol. 2015, 44, 1889-1899. [CrossRef] [PubMed]

36. Lim, S.; Ha, M.; Hwang, S.-S.; Son, M.; Kwon, H.-J. Disparities in Children's Blood Lead and Mercury Levels According to Community and Individual Socioeconomic Positions. Int. J. Environ. Res. Public Health 2015, 12, 6232-6248. [CrossRef]

37. Wehby, G.L.; Prater, K.; McCarthy, A.M.; Castilla, E.E.; Murray, J.C. The Impact of Maternal Smoking during Pregnancy on Early Child Neurodevelopment. J. Hum. Cap. 2011, 5, 207-254. [CrossRef] [PubMed]

38. Gaxiola-Robles, R.; Bentzen, R.; Zenteno-Savín, T.; Labrada-Martagón, V.; Castellini, J.M.; Celis, A.; O’Hara, T.; Méndez-Rodríguez, L.C. Marine diet and tobacco exposure affects mercury concentrations in pregnant women (I) from Baja California Sur, Mexico. Toxicol. Rep. 2014, 1, 1123-1132. [CrossRef] [PubMed]

39. Avella-Garcia, C.B.; Julvez, J. Seafood Intake and Neurodevelopment: A Systematic Review. Curr. Environ. Health Rep. 2014, 1, 46-77. [CrossRef]

40. Moon, S.-W.; Gwak, J.-I.; Park, Y.-H. The Effect of Smoking and Second-Hand Smoking on the Concentration of Mercury, Lead and Cadmium in the Blood: Based on the Fifth Korea National Health and Nutrition Examination Survey. Korean J. Fam. Pract. 2016, 6, 44-48. [CrossRef]

41. Næss, S.; Kjellevold, M.; Dahl, L.; Nerhus, I.; Midtbø, L.K.; Bank, M.S.; Rasinger, J.D.; Markhus, M.W. Effects of seafood consumption on mercury exposure in Norwegian pregnant women: A randomized controlled trial. Environ. Int. 2020, 141, 105759. [CrossRef]

42. Sharp, M.; DeMauro, S.B. Counterbalanced Comparison of the BSID-II and Bayley-III at Eighteen to Twenty-two Months Corrected Age. J. Dev. Behav. Pediatr. 2017, 38, 322-329. [CrossRef]

43. Suzuki, K.; Nakai, K.; Sugawara, T.; Nakamura, T.; Ohba, T.; Shimada, M.; Hosokawa, T.; Okamura, K.; Sakai, T.; Kurokawa, N.; et al. Neurobehavioral effects of prenatal exposure to methylmercury and PCBs, and seafood intake: Neonatal behavioral assessment scale results of Tohoku study of child development. Environ. Res. 2010, 110, 699-704. [CrossRef]

44. Rothenberg, S.E.; Yu, X.; Liu, J.; Biasini, F.J.; Hong, C.; Jiang, X.; Nong, Y.; Cheng, Y.; Korrick, S.A. Maternal methylmercury exposure through rice ingestion and offspring neurodevelopment: A prospective cohort study. Int. J. Hyg. Environ. Health 2016, 219, 832-842. [CrossRef] [PubMed]

45. Marques, R.C.; Dórea, J.G.; Bernardi, J.V.; Bastos, W.R.; Malm, O. Prenatal and postnatal mercury exposure, breastfeeding and neurodevelopment during the first 5 years. Cogn. Behav. Neurol. 2009, 22, 134-141. [CrossRef] [PubMed]

46. Nyanza, E.C.; Bernier, F.P.; Martin, J.W.; Manyama, M.; Hatfield, J.; Dewey, D. Effects of prenatal exposure and co-exposure to metallic or metalloid elements on early infant neurodevelopmental outcomes in areas with small-scale gold mining activities in Northern Tanzania. Environ. Int. 2021, 149, 106104. [CrossRef] [PubMed]

47. Xu, Y.; Khoury, J.C.; Sucharew, H.; Dietrich, K.; Yolton, K. Low-level gestational exposure to mercury and maternal fish consumption: Associations with neurobehavior in early infancy. Neurotoxicol. Teratol. 2016, 54, 61-67. [CrossRef]

48. Kim, Y.; Ha, E.-H.; Park, H.; Ha, M.; Kim, Y.; Hong, Y.-C.; Lee, E.J.; Kim, H.; Chang, N.; Kim, B.-N. Prenatal mercury exposure, fish intake and neurocognitive development during first three years of life: Prospective cohort mothers and Children's environmental health (MOCEH) study. Sci. Total Environ. 2018, 615, 1192-1198. [CrossRef]

49. Polanska, K.; Hanke, W.; Sobala, W.; Trzcinka-Ochocka, M.; Ligocka, D.; Brzeznicki, S.; Strugala-Stawik, H.; Magnus, P. Developmental Effects of Exposures to Environmental Factors: The Polish Mother and Child Cohort Study. Biomed. Res. Int. 2013, 2013, 629716. [CrossRef]

50. Davidson, P.W.; Strain, J.J.; Myers, G.J.; Thurston, S.W.; Bonham, M.P.; Shamlaye, C.F.; Stokes-Riner, A.; Wallace, J.M.; Robson, P.J.; Duffy, E.M.; et al. Neurodevelopmental effects of maternal nutritional status and exposure to methylmercury from eating fish during pregnancy. Neurotoxicology 2008, 29, 767-775. [CrossRef]

51. Lin, C.C.; Chen, Y.C.; Su, F.C.; Lin, C.M.; Liao, H.F.; Hwang, Y.H.; Hsieh, W.S.; Jeng, S.F.; Su, Y.N.; Chen, P.C. In utero exposure to environmental lead and manganese and neurodevelopment at 2 years of age. Environ. Res 2013, 123, 52-57. [CrossRef]

52. Johnson, S.; Marlow, N. Developmental screen or developmental testing? Early Hum. Dev. 2006, 82, 173-183. [CrossRef]

53. Shah-Kulkarni, S.; Lee, S.; Jeong, K.S.; Hong, Y.-C.; Park, H.; Ha, M.; Kim, Y.; Ha, E.-H. Prenatal exposure to mixtures of heavy metals and neurodevelopment in infants at 6 months. Environ. Res. 2020, 182, 109122. [CrossRef] [PubMed] 
54. Strain, J.J.; Yeates, A.J.; van Wijngaarden, E.; Thurston, S.W.; Mulhern, M.S.; McSorley, E.M.; Watson, G.E.; Love, T.M.; Smith, T.H.; Yost, K.; et al. Prenatal exposure to methyl mercury from fish consumption and polyunsaturated fatty acids: Associations with child development at 20 mo of age in an observational study in the Republic of Seychelles. Am. J. Clin. Nutr. 2015, 101, 530-537. [CrossRef]

55. Boucher, O.; Muckle, G.; Jacobson, J.L.; Carter, R.C.; Kaplan-Estrin, M.; Ayotte, P.; Dewailly, É.; Jacobson, S.W. Domain-specific effects of prenatal exposure to PCBs, mercury, and lead on infant cognition: Results from the Environmental Contaminants and Child Development Study in Nunavik. Environ. Health Perspect. 2014, 122, 310-316. [CrossRef]

56. Jedrychowski, W.; Perera, F.; Jankowski, J.; Rauh, V.; Flak, E.; Caldwell, K.L.; Jones, R.L.; Pac, A.; Lisowska-Miszczyk, I. Fish consumption in pregnancy, cord blood mercury level and cognitive and psychomotor development of infants followed over the first three years of life: Krakow epidemiologic study. Environ. Int. 2007, 33, 1057-1062. [CrossRef] [PubMed]

57. Kim, S.; Eom, S.; Kim, H.J.; Lee, J.J.; Choi, G.; Choi, S.; Kim, S.; Kim, S.Y.; Cho, G.; Kim, Y.D.; et al. Association between maternal exposure to major phthalates, heavy metals, and persistent organic pollutants, and the neurodevelopmental performances of their children at 1 to 2years of age- CHECK cohort study. Sci. Total Environ. 2018, 624, 377-384. [CrossRef]

58. Lederman, S.A.; Jones, R.L.; Caldwell, K.L.; Rauh, V.; Sheets, S.E.; Tang, D.; Viswanathan, S.; Becker, M.; Stein, J.L.; Wang, R.Y.; et al. Relation between cord blood mercury levels and early child development in a World Trade Center cohort. Environ. Health Perspect. 2008, 116, 1085-1091. [CrossRef] [PubMed]

59. Llop, S.; Guxens, M.; Murcia, M.; Lertxundi, A.; Ramon, R.; Riano, I.; Rebagliato, M.; Ibarluzea, J.; Tardon, A.; Sunyer, J.; et al. Prenatal exposure to mercury and infant neurodevelopment in a multicenter cohort in Spain: Study of potential modifiers. Am. J. Epidemiol. 2012, 175, 451-465. [CrossRef]

60. Yi, Y.G.; Sung, I.Y.; Yuk, J.S. Comparison of Second and Third Editions of the Bayley Scales in Children with Suspected Developmental Delay. Ann. Rehabil. Med. 2018, 42, 313-320. [CrossRef]

61. Barbone, F.; Rosolen, V.; Mariuz, M.; Parpinel, M.; Casetta, A.; Sammartano, F.; Ronfani, L.; Vecchi Brumatti, L.; Bin, M.; Castriotta, L.; et al. Prenatal mercury exposure and child neurodevelopment outcomes at 18 months: Results from the Mediterranean PHIME cohort. Int. J. Hyg. Environ. Health 2019, 222, 9-21. [CrossRef]

62. Castriotta, L.; Rosolen, V.; Biggeri, A.; Ronfani, L.; Catelan, D.; Mariuz, M.; Bin, M.; Brumatti, L.V.; Horvat, M.; Barbone, F. The role of mercury, selenium and the Se-Hg antagonism on cognitive neurodevelopment: A 40-month follow-up of the Italian mother-child PHIME cohort. Int. J. Hyg. Environ. Health 2020, 230, 113604. [CrossRef]

63. Nišević, J.R.; Prpić, I.; Kolić, I.; Baždarić, K.; Tratnik, J.S.; Prpić, I.; Mazej, D.; Špirić, Z.; Barbone, F.; Horvat, M. Combined prenatal exposure to mercury and LCPUFA on newborn's brain measures and neurodevelopment at the age of 18 months. Environ. Res. 2019, 178, 108682. [CrossRef] [PubMed]

64. Snoj Tratnik, J.; Falnoga, I.; Trdin, A.; Mazej, D.; Fajon, V.; Miklavčič, A.; Kobal, A.B.; Osredkar, J.; Sešek Briški, A.; Krsnik, M.; et al. Prenatal mercury exposure, neurodevelopment and apolipoprotein E genetic polymorphism. Environ. Res. 2017, 152, 375-385. [CrossRef] [PubMed]

65. Valent, F.; Mariuz, M.; Bin, M.; Little, D.; Mazej, D.; Tognin, V.; Tratnik, J.; McAfee, A.J.; Mulhern, M.S.; Parpinel, M.; et al. Associations of prenatal mercury exposure from maternal fish consumption and polyunsaturated fatty acids with child neurodevelopment: A prospective cohort study in Italy. J. Epidemiol. 2013, 23, 360-370. [CrossRef] [PubMed]

66. Wang, J.; Wu, W.; Li, H.; Cao, L.; Wu, M.; Liu, J.; Gao, Z.; Zhou, C.; Liu, J.; Yan, C. Relation of prenatal low-level mercury exposure with early child neurobehavioral development and exploration of the effects of sex and DHA on it. Environ. Int. 2019, 126, 14-23. [CrossRef] [PubMed]

67. Tatsuta, N.; Nakai, K.; Murata, K.; Suzuki, K.; Iwai-Shimada, M.; Kurokawa, N.; Hosokawa, T.; Satoh, H. Impacts of prenatal exposures to polychlorinated biphenyls, methylmercury, and lead on intellectual ability of 42-month-old children in Japan. Environ. Res. 2014, 133, 321-326. [CrossRef]

68. Freire, C.; Amaya, E.; Gil, F.; Fernández, M.F.; Murcia, M.; Llop, S.; Andiarena, A.; Aurrekoetxea, J.; Bustamante, M.; Guxens, M.; et al. Prenatal co-exposure to neurotoxic metals and neurodevelopment in preschool children: The Environment and Childhood (INMA) Project. Sci. Total Environ. 2018, 621, 340-351. [CrossRef]

69. Oken, E.; Radesky, J.S.; Wright, R.O.; Bellinger, D.C.; Amarasiriwardena, C.J.; Kleinman, K.P.; Hu, H.; Gillman, M.W. Maternal Fish Intake during Pregnancy, Blood Mercury Levels, and Child Cognition at Age 3 Years in a US Cohort. Am. J. Epidemiol. 2008, 167, 1171-1181. [CrossRef]

70. Oken, E.; Wright, R.O.; Kleinman, K.P.; Bellinger, D.; Amarasiriwardena, C.J.; Hu, H.; Rich-Edwards, J.W.; Gillman, M.W. Maternal fish consumption, hair mercury, and infant cognition in a U.S. Cohort. Environ. Health Perspect. 2005, 113, 1376-1380. [CrossRef]

71. Tatsuta, N.; Murata, K.; Iwai-Shimada, M.; Yaginuma-Sakurai, K.; Satoh, H.; Nakai, K. Psychomotor Ability in Children Prenatally Exposed to Methylmercury: The 18-Month Follow-Up of Tohoku Study of Child Development. Tohoku J. Exp. Med. 2017, 242, 1-8. [CrossRef]

72. Prpic, I.; Milardovic, A.; Vlasic-Cicvaric, I.; Spiric, Z.; Radic Nisevic, J.; Vukelic, P.; Snoj Tratnik, J.; Mazej, D.; Horvat, M. Prenatal exposure to low-level methylmercury alters the child's fine motor skills at the age of 18 months. Environ. Res. 2017, 152, 369-374. [CrossRef]

73. Hu, Y.; Chen, L.; Wang, C.; Zhou, Y.; Zhang, Y.; Wang, Y.; Shi, R.; Gao, Y.; Tian, Y. Prenatal low-level mercury exposure and infant neurodevelopment at 12 months in rural northern China. Environ. Sci. Pollut. Res. Int. 2016, 23, 12050-12059. [CrossRef] [PubMed] 
74. Daniels, J.L.; Longnecker, M.P.; Rowland, A.S.; Golding, J. Fish intake during pregnancy and early cognitive development of offspring. Epidemiology 2004, 15, 394-402. [CrossRef] [PubMed]

75. Wu, J.; Ying, T.; Shen, Z.; Wang, H. Effect of low-level prenatal mercury exposure on neonate neurobehavioral development in China. Pediatr. Neurol. 2014, 51, 93-99. [CrossRef] [PubMed]

76. Golding, J.; Gregory, S.; Iles-Caven, Y.; Hibbeln, J.; Emond, A.; Taylor, C.M. Associations between prenatal mercury exposure and early child development in the ALSPAC study. Neurotoxicology 2016, 53, 215-222. [CrossRef]

77. Valko, M.; Morris, H.; Cronin, M.T. Metals, toxicity and oxidative stress. Curr. Med. Chem. 2005, 12, 1161-1208. [CrossRef]

78. Chapman, L.; Chan, H.M. The influence of nutrition on methyl mercury intoxication. Environ. Health Perspect. 2000, 108, 29-56. [CrossRef]

79. Philibert, A.; Fillion, M.; Mergler, D. Mercury exposure and premature mortality in the Grassy Narrows First Nation community: A retrospective longitudinal study. Lancet Planet. Health 2020, 4, e141-e148. [CrossRef]

80. Grandjean, P.; Weihe, P.; White, R.F.; Debes, F.; Araki, S.; Yokoyama, K.; Murata, K.; Sørensen, N.; Dahl, R.; Jørgensen, P.J. Cognitive Deficit in 7-Year-Old Children with Prenatal Exposure to Methylmercury. Neurotoxicol. Teratol. 1997, 19, 417-428. [CrossRef]

81. Taylor, C.M.; Golding, J.; Emond, A.M. Lead, cadmium and mercury levels in pregnancy: The need for international consensus on levels of concern. J. Dev. Orig. Health Dis. 2014, 5, 16-30. [CrossRef]

82. Rice, D.C.; Schoeny, R.; Mahaffey, K. Methods and rationale for derivation of a reference dose for methylmercury by the US EPA. Risk Anal. Int. J. 2003, 23, 107-115. [CrossRef]

83. Rice, D.C. The US EPA reference dose for methylmercury: Sources of uncertainty. Environ. Res. 2004, 95, 406-413. [CrossRef] [PubMed]

84. Mahaffey, K.R.; Clickner, R.P.; Bodurow, C.C. Blood organic mercury and dietary mercury intake: National Health and Nutrition Examination Survey, 1999 and 2000. Environ. Health Perspect. 2004, 112, 562-570. [CrossRef] [PubMed]

85. Alhibshi, E. Subclinical neurotoxicity of mercury: A behavioural, molecular mechanisms and therapeutic perspective. Res. J. Pharm. Biol. Chem. Sci. 2012, 3, 34-42.

86. Taylor, C.M.; Emmett, P.M.; Emond, A.M.; Golding, J. A review of guidance on fish consumption in pregnancy: Is it fit for purpose? Public Health Nutr. 2018, 21, 2149-2159. [CrossRef] [PubMed]

87. Saavedra, S.; Fernández-Recamales, Á.; Sayago, A.; Cervera-Barajas, A.; González-Domínguez, R.; Gonzalez-Sanz, J.D. Impact of dietary mercury intake during pregnancy on the health of neonates and children: A systematic review. Nutr. Rev. 2022, 80, 317-328. [CrossRef]

88. Hibbeln, J.R.; Spiller, P.; Brenna, J.T.; Golding, J.; Holub, B.J.; Harris, W.S.; Kris-Etherton, P.; Lands, B.; Connor, S.L.; Myers, G.; et al. Relationships between seafood consumption during pregnancy and childhood and neurocognitive development: Two systematic reviews. Prostaglandins Leukot. Essent. Fat. Acids 2019, 151, 14-36. [CrossRef]

89. Grandjean, P.; Budtz-Jørgensen, E.; Jørgensen, P.J.; Weihe, P. Umbilical cord mercury concentration as biomarker of prenatal exposure to methylmercury. Environ. Health Perspect. 2005, 113, 905-908. [CrossRef]

90. Kozikowska, I.; Binkowski, Ł.J.; Szczepańska, K.; Sławska, H.; Miszczuk, K.; Śliwińska, M.; Łaciak, T.; Stawarz, R. Mercury concentrations in human placenta, umbilical cord, cord blood and amniotic fluid and their relations with body parameters of newborns. Environ. Pollut. 2013, 182, 256-262. [CrossRef] 